\title{
Effect and mechanism of total alkaloids of strychnine on papain induced rabbit knee osteoarthritis.
}

\author{
Zhenqiang Hong*', Hongjian Gao, Youxin Su, Bo Xu, Zijian Wu \\ College of Traditional Chinese Medicine, Fujian University of Traditional Chinese Medicine, Fujian, Fuzhou, PR China
}

\begin{abstract}
Objective: To examine the effect and mechanism of Strychnine total alkaloids on a model of knee osteoarthritis.

Methods: New Zealand rabbits were randomly divided into normal group, model group, total alkaloid high/medium/low dose groups and a sodium hyaluronate group. Except for the normal control group, all other groups were injected with papain in the joint cavity to establish osteoarthritis. After 8 weeks of modeling, a high, medium and low dose of Strychnine total alkaline was given by injection in the joint cavity, $0.3 \mathrm{ml}, 0.2 \mathrm{ml}$, and $0.1 \mathrm{ml}$ respectively. At this time an intra-articular injection of $0.2 \mathrm{ml}$ sodium hyaluronate was also given to the hyaluronate group. The injection of Strychnine total alkaline was continually administered, twice a week for 5 weeks. All animals were harvested for analysis one week after the final dose. Pathological changes of articular cartilage were observed and evaluated by Mankin's score. Total blood viscosity (low shear, middle shear, high shear) and plasma viscosity were measured by hemorheometer. The levels of IL-1 and IL-6, SOD, LPO, NO, and TNF in the synovial fluid and PYD in urine were additionally measured by enzyme-linked immunosorbent assay (ELISA).

Main findings: Compared with the osteoarthritis controls, the cartilage injury was significantly improved in the total alkaloid group. Mankin's score was significantly decreased $(\mathbf{P}<0.05)$, and the blood viscosity and plasma viscosity of low, middle and high shear rates was decreased $(\mathbf{P}<0.05)$. Finally, IL-1, IL-6, LPO, NO and TNF in synovial fluid were decreased while SOD content increased $(P<0.05)$.

Conclusion: These results indicate that Strychnine total alkaloids have reparative effects on cartilage injury during osteoarthritis. The mechanisms may at least partially be related to the inhibition of inflammatory factors, the regulation of free radicals and the overall improvement of cartilage metabolism.
\end{abstract}

Keywords: Strychnine total alkaloids, Rabbit knee osteoarthritis, Papain model, Mechanism of action.

\section{Abbreviations:}

ELISA: Enzyme-Linked Immunosorbent Assay; EMA: European Medicines Agency; HA: Hyaluronic Acid; IL-1: Interleukin-1; IL-6: Interleukin-6; KOA: Knee Osteoarthritis; LPO: Lipid Peroxide; MSCs: Mesenchymal Stem Cells; NO: Nitric Oxide; NSAIDs: Non-Steroidal Anti-Inflammatory
Drugs; OA: Osteoarthritis; OARSI: Osteoarthritis Research Society International; PRP: Platelet Rich Plasma; PYD: Pyridinoline; SOD: Superoxide Dismutase; TNF: Tumor Necrosis Factor; US FDA: United States Food and Drug Administration.

\section{Introduction}

Osteoarthritis (OA) is chronic degenerative osteoarthropathy characterized by joint pain, stiffness, limitation of flexion, extension and walking, and even joint deformity and disability which is difficult to be treated, and there is no effective drugs and methods that can prevent the progression of this disease. Modern medical treatment of osteoarthritis includes drugs and surgery, which give priority to non-steroidal anti-inflammatory drugs. These drugs can relieve pain but was unable to repair the damage of articular cartilage and stop the development of OA. Long-term use of these drugs will also cause gastric ulcer, stomach bleeding and liver and kidney damage and other side effects. Surgical treatment can effectively relieve pain in the hip or knee joint, but it is costly and traumatic with some serious complications such as infection, deep-vein thrombosis, prosthesis loosening, etc. Intra-articular drug injection has been widely applied to the treatment of osteoarthritis in recent years, most of which promote cartilage regeneration and delay degeneration $[1,2]$. Due to the direct effect and rapid onset, intra-articular drug injection has become more and more popular in OA patients [3]. Traditional Chinese Medicine in the treatment of osteoarthritis focus on how to protect cartilage damage of joint tissue repair and can prevent further development of pathological condition. These traditional 
Chinese medical treatments have the advantages of reliable curative effect, low cost and less adverse reaction.

Strychnos are dry seeds for the Strychnos pierriana A. W. Hill., which is one of the commonly used traditional Chinese medicines for arthritis treatment. The compound has been associated with the promotion of blood circulation, pain relief, and the elimination of mass swelling. Strychnine alkaloids are the main chemical composition of the substance. Studies have previously shown that intravenous injection of Strychnine alkaloids can promote the repair of cartilage injury, though its mechanisms are unknown [4]. The pathogenesis of osteoarthritis is complex, and its pathogenic factors are mainly related to inflammatory factors, free radicals, intraosseous pressure and so on. Therefore, in this study, a papain model of rabbit knee osteoarthritis was established and the total alkaloids of Strychnosum were injected into joint cavity to observe the effects of the compound on the pathology and hemorheology of articular cartilage. Additionally, we sought to explore the effects of Strychnonine on inflammatory factors, free radicals and markers of intraosseous pressure, including IL-1 and IL 6, SOD, LPO, NO, TNF and urinary PYD.

\section{Materials and Methods}

\section{Drugs and reagents}

Papain, Lot 107147 (Sigma, USA); Strychnine total alkaloid injection solution, $1 \mathrm{ml} / \mathrm{each}$ (pre-preparation by the research group); sodium hyaluronate injection solution, $20 \mathrm{mg} / \mathrm{branch}$ (Shanghai Haohai Biotechnology); IL-1, IL-6, SOD, LPO, NO, TNF, PYD enzyme-linked immunosorbent assay reagent (Shanghai Xitang Biotechnology Co., Ltd.) were used in this study.

\section{Animals}

Forty eight healthy New Zealand rabbits (body weight 2.5 3.5 $\mathrm{kg}$, male (24) and female (24)) were provided by the Shanghai Songjiang District Songlian experimental animal company, license number: SCXK (Shanghai) 2012-0011. Fujian University of Traditional Chinese Medicine Experimental Animal Center provided clean medical laboratory animal environmental facilities; license number SCXK (Min) 2012-0001. All animal protocols were approved by the Animal Care Committee of Fujian University of Traditional Chinese Medicine.

\section{Animal model}

Except for the normal control group, all other groups received papain injection into the joint cavity to establish the osteoarthritis model. Both the left and right knee was randomly selected for treatment. Anesthesia was administered via the ear vein (pentobarbital sodium, $30 \mathrm{mg} / \mathrm{kg}$ ). The rabbit was supine fixed with skin preparation and routine disinfection. The rabbit knee joint was mildly bent and $0.5 \mathrm{ml}$ of $1.6 \%$ papain saline solution was injected into the knee joint cavity through the patellar ligament. The needle was injected through the middle or both sides of the joint. The papain injection was performed once every $3 \mathrm{~d}$ for a total of three continuous doses. During osteoarthritis modeling, rabbits were forced into daily activity for at least $30 \mathrm{~min}$. The duration of activity was separated into 2 episodes which lasted for 4 weeks.

\section{Dose and method}

After 8 weeks of disease modeling, the high, medium and low dose total alkaloid groups were given $0.3 \mathrm{ml}, 0.2 \mathrm{ml}, 0.1 \mathrm{ml}$ of total alkaloids respectively; $0.2 \mathrm{ml}$ of sodium hyaluronate was additionally given to the sodium hyaluronate group (Table 1). Injections were performed in each joint cavity, twice a week for 5 weeks. A sham and model control group were routinely raised, with no other special treatment. One week after administration of the final dose, all animals were sacrificed.

Table 1. Animal groups.

\begin{tabular}{|c|c|c|c|}
\hline Group & $\mathbf{n}$ & Drugs & Dose \\
\hline Normal control & 8 & $\mathrm{ddH}_{2} \mathrm{O}$ & $0.2 \mathrm{ml}$ \\
\hline Model control & 8 & $\mathrm{dd}_{2} \mathrm{O}$ & $0.2 \mathrm{ml}$ \\
\hline $\begin{array}{l}\text { Strychnine total alkaloids } \\
\text { high dose }\end{array}$ & 8 & $\begin{array}{l}\text { Strychnine total } \\
\text { alkaloids }\end{array}$ & $0.3 \mathrm{ml}$ \\
\hline $\begin{array}{l}\text { Strychnine total alkaloids } \\
\text { medium dose }\end{array}$ & 8 & $\begin{array}{l}\text { Strychnine total } \\
\text { alkaloids }\end{array}$ & $0.2 \mathrm{ml}$ \\
\hline $\begin{array}{l}\text { Strychnine total alkaloids low } \\
\text { dose }\end{array}$ & 8 & $\begin{array}{l}\text { Strychnine total } \\
\text { alkaloids }\end{array}$ & $0.1 \mathrm{ml}$ \\
\hline Sodium hyaluronate & 8 & $\begin{array}{l}\text { sodium } \\
\text { hyaluronate }\end{array}$ & $0.2 \mathrm{ml}$ \\
\hline
\end{tabular}

\section{Collection and preparation of specimens}

Animals were fasted for $10 \mathrm{~h}$ prior to harvest. Blood samples were taken from the ear vein of the rabbit, and put into the anticoagulation tube. Immediately after shaking, the blood rheology was measured. A longitudinal incision was made along the inner edge of the patella, next separation of the joint capsule was made layer by layer.

Repeated flexion and extension of the joints was forced in order to squeeze the joint fluid to the medial side of the patellar capsule, after which the joint capsule was gently opened and $1.5 \mathrm{ml}$ of $0.9 \%$ sodium chloride was injected. Withdrawal of the joint fluid was then swiftly performed and high speed centrifugation was carried out for $15 \mathrm{~min}\left(4^{\circ} \mathrm{C}, 15000 \mathrm{r} \cdot \mathrm{min}\right)$ to extract the supernatant, stored in the refrigerator at $-80^{\circ} \mathrm{C}$.

Finally the knee joint capsule was cut, followed by the rapid removal of the femoral condylar articular cartilage subsequently chopped into $0.5 \times 0.5 \times 0.5 \mathrm{~cm}^{3}$ tissue blocks. Specimens were fixed in $40 \mathrm{~g} / \mathrm{L}$ paraformaldehyde, $10 \%$ EDTA-2Na de-calcified for 8 weeks and paraffin embedded. Observation of pathological changes of cartilage tissue were performed by Hematoxylin-eosin staining. 


\section{Pathological changes of articular cartilage and Mankin's score}

Femoral condyle cartilage surface, the number of chondrocytes and the level of chondrocytes, cartilage polysaccharide staining and tidal line integrity were observed by optical microscopy. Mankin's score was used to evaluate cartilage injury [5]. Scoring criteria are shown in Table 2. Blood rheometer was used to detect whole blood viscosity (low shear, middle cut, high cut) and plasma viscosity. The expression of inflammatory and free radical markers IL-1, IL-6, SOD, LPO, NO, and TNF were performed in synovial fluid and PYD assessment was made in urine. All markers were detected by enzyme-linked immunosorbent assay (ELISA).

Table 2. Mankin's score criteria (Total score 14).

\begin{tabular}{|c|c|c|c|c|c|c|c|}
\hline Cartilage structure & & Chondrocyte & & Proteoglycans stained & & Tide line integrity & \\
\hline Characteristic & Score & Characteristic & Score & Characteristic & Score & Characteristic & Score \\
\hline Normal & 0 & Normal & 0 & Normal & 0 & Normal & 0 \\
\hline $\begin{array}{l}\text { Superficial cartilage layers } \\
\text { small crack }\end{array}$ & 1 & Diffuse increase & 1 & $\begin{array}{l}\text { Dyeing } \\
\text { diminishes }\end{array}$ & 1 & Multiple tide line & 1 \\
\hline Crack reach transition layer & 2 & $\begin{array}{l}\text { Large number of clusters of } \\
\text { cells }\end{array}$ & 2 & $\begin{array}{l}\text { Dyeing moderately } \\
\text { diminishes }\end{array}$ & 2 & $\begin{array}{l}\text { Vascular invasion of the } \\
\text { tide line }\end{array}$ & 2 \\
\hline Crack reach radiation layer & 3 & $\begin{array}{l}\text { The number is significantly } \\
\text { reduced }\end{array}$ & 3 & $\begin{array}{l}\text { Dyeing significantly } \\
\text { diminishes }\end{array}$ & 3 & & \\
\hline $\begin{array}{l}\text { Crack reach calcification } \\
\text { layer }\end{array}$ & 4 & & & $\begin{array}{l}\text { Dyeing completely } \\
\text { diminishes }\end{array}$ & 4 & & \\
\hline
\end{tabular}

\section{Statistical analysis}

Statistical analysis was performed with SPSS18.0 software (Company, Location). The data were expressed as $(\overline{\mathrm{x}} \pm \mathrm{s})$; ANOVA or t-test was used where appropriate. The Pearson simple correlation coefficient was additionally analyzed. $\mathrm{P}<0.05$ denoted statistically significant differences among groups.

\section{Results}

\section{The pathological changes of articular cartilage}

In the normal control group, articular cartilage surface was smooth, with a four-layer structure (from superfice to deepest layer: surface layer, transitional stratum, radiated stratum, and calcification layer) clearly distinguishable, and with the integrated tidemark visible. The adhesion line was also obvious in this group. Surface cells appeared smaller, flattened, and parallel to the surface of the cartilage. The transitional cells were larger, rounded or elliptical. Radiation cells tended to be arranged in column, evenly distributed and neatly arranged while calcified cells scattered in distribution. In the osteoarthritis group, the articular cartilage surface was not smooth. Fewer cells were apparent in the surface and radiation layers. Additionally, cells in the transitional layer were unevenly distributed and the four-layer structure was not easily distinguished. Finally, the tide line was not obvious and complete in this group. Generally, the Strychnine total alkaloids groups appeared significantly repaired at the articular cartilage, especially in the high dose group. These groups manifested a smooth cartilage surface, and a distinguishable four-layer structure.
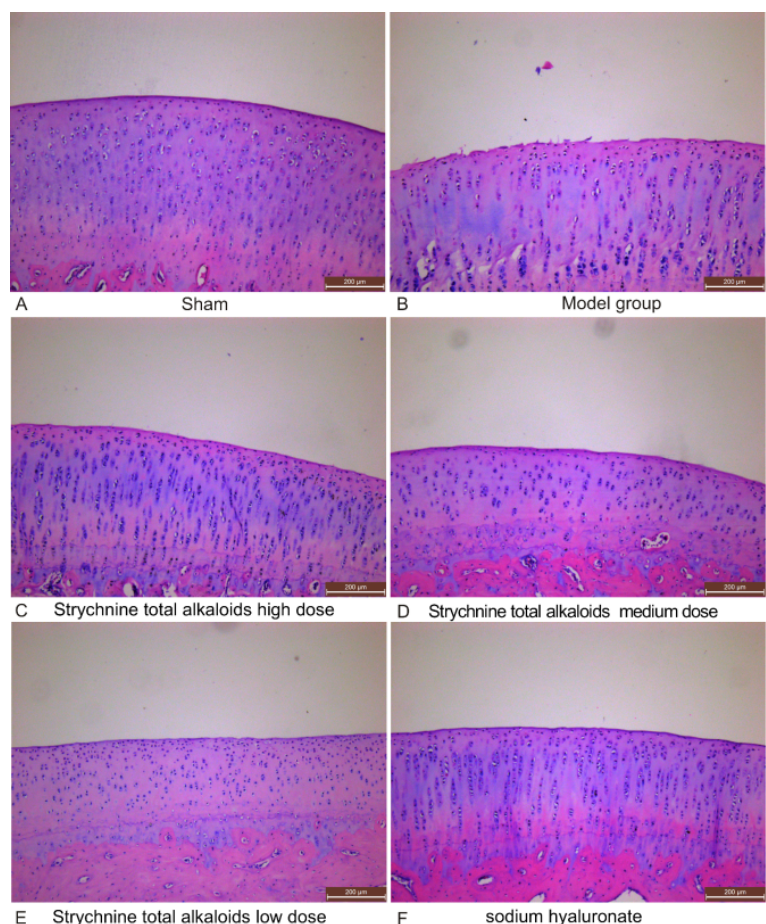

E Strychnine total alkaloids low dos

sodium hyaluronate

Figure 1. Observation of articular cartilage pathology by light microscopy (paraffin section-HE staining). (A) Normal control; (B) Model control; (C) Strychnine total alkaloids high dose; (D) Strychnine total alkaloids medium dose; (E) Strychnine total alkaloids low dose; (F) Sodium hyaluronate Scale bar: $200 \mu \mathrm{m}$. 
The number of chondrocytes in all layers was increased and more evenly distributed. In the sodium hyaluronate group, articular cartilage injury was also significantly repaired compared to the disease model group, displaying a smooth cartilage surface, a restored number of cell layers, and the evenly distributed, columnar arrangement obvious in the radiation layer. In the group the tide line was also seemingly complete (Figure 1).

\section{Articular cartilage Mankin's score}

The score in osteoarthritis model group was significantly higher than that in normal control group $(\mathrm{P}<0.05)$. The score in total alkaloid groups were significantly lower than that of the model group $(\mathrm{P}<0.05)$. The medium dose group was the most significant. The score in sodium hyaluronate group was significantly lower than that in model group $(\mathrm{P}<0.05)$. There was no significant difference between the medium dose group and the sodium hyaluronate group $(\mathrm{P}>0.05$ and Table 3$)$.

Table 3. Articular cartilage Mankin's score.

\begin{tabular}{ll}
\hline Groups & Score \\
\hline Normal control & $0.25 \pm 0.16$ \\
\hline Model & $8 \pm 0.18^{\mathrm{a}}$ \\
\hline Strychnine total alkaloids high dose & $6 \pm 0.19^{\mathrm{b}}$ \\
\hline
\end{tabular}

Table 4. Whole blood and plasma viscosity.

\begin{tabular}{lc}
\hline Strychnine total alkaloids medium dose & $4.25 \pm 0.16^{\mathrm{b}}$ \\
\hline Strychnine total alkaloids low dose & $6 \pm 0.18$ \\
\hline Sodium hyaluronate & $4.13 \pm 0.13^{\mathrm{b}}$ \\
\hline $\begin{array}{l}\text { Note: Compared with normal group, ap<0.05; Compared with model group, } \\
\text { bP<0.05. }\end{array}$
\end{tabular}

\section{Whole blood and plasma viscosity}

Compared with the normal control group, the low, medium and high shear whole blood viscosity and plasma viscosity of the osteoarthritis model group were significantly higher $(\mathrm{P}<0.05)$. Compared with the disease model group, the low, medium and high shear blood viscosity and plasma viscosity of the total alkaloid high, middle and low dose groups were comparably decreased $(\mathrm{P}<0.05)$, with the low, medium and high shear blood viscosity and plasma viscosity of the sodium hyaluronate group similarly reduced $(\mathrm{P}<0.05)$. Within the total alkaloid groups, the low dose group low, medium and high shear blood viscosity and plasma viscosity were significantly lower than the high and middle dose groups $(\mathrm{P}<0.05)$, demonstrating a dose-dependent correlation between total alkaloid and blood and plasma viscosity. On the other hand, there was no significant difference in blood viscosity and plasma viscosity between the high and middle dose groups or the sodium hyaluronate groups ( $\mathrm{P}>0.05$ and Table 4$)$.

\begin{tabular}{|c|c|c|c|c|c|}
\hline \multirow[t]{2}{*}{ Groups } & \multicolumn{3}{|c|}{ The whole blood viscosity (mPa.s) } & \multirow{2}{*}{\multicolumn{2}{|c|}{$\begin{array}{l}\text { Plasma } \\
\text { (mPa.s) }\end{array}$}} \\
\hline & Low shear (10 s) & Medium shear (60 s) & High shear (150 s) & & \\
\hline Normal & $7.65 \pm 0.25$ & $5.80 \pm 0.12$ & $4.16 \pm 0.09$ & $1.40 \pm 0.02$ & \\
\hline Model & $13.51 \pm 0.21^{a}$ & $11.31 \pm 0.29^{a}$ & $6.10 \pm 0.21^{a}$ & $2.22 \pm 0.13^{a}$ & \\
\hline Strychnine total alkaloids high dose & $9.77 \pm 0.29^{b}$ & $7.67 \pm 0.15^{b}$ & $4.74 \pm 0.12^{\mathrm{b}}$ & $1.65 \pm 0.05^{b}$ & \\
\hline Strychnine total alkaloids medium dose & $9.94 \pm 0.07^{\mathrm{b}}$ & $7.63 \pm 0.16^{b}$ & $4.71 \pm 0.10^{\mathrm{b}}$ & $1.67 \pm 0.13^{b}$ & \\
\hline Strychnine total alkaloids low dose & $10.31 \pm 0.09^{b}$ & $8.17 \pm 0.11^{b}$ & $4.92 \pm 0.13^{b}$ & $1.94 \pm 0.16^{b}$ & \\
\hline Sodium hyaluronate & $9.75 \pm 0.12^{\mathrm{b}}$ & $7.34 \pm 0.13^{b}$ & $4.54 \pm 0.20^{b}$ & $1.55 \pm 0.06^{b}$ & \\
\hline
\end{tabular}

Note: Compared with normal group, aP $<0.05$; Compared with model group, ${ }^{\mathrm{b}} \mathrm{P}<0.05$.

Table 5. The comparative expression of inflammatory and free radical markers.

\begin{tabular}{|c|c|c|c|c|c|c|c|}
\hline \multirow[t]{2}{*}{ Group } & \multicolumn{6}{|l|}{ Synovial fluid } & \multirow{2}{*}{$\begin{array}{l}\text { Urine } \\
\text { PYD (ng/ml) }\end{array}$} \\
\hline & IL-1 (Pg/ml) & IL-6 (Pg/ml) & SOD (u/ml) & LPO (nmol/ml) & $\mathrm{NO}(\mu \mathrm{mol} / \mathrm{L})$ & TNF (Pg/ml) & \\
\hline Normal & $5.61 \pm 0.99$ & $8.63 \pm 0.76$ & $71.74 \pm 1.93$ & $83.60 \pm 2.64$ & $55.84 \pm 2.78$ & 0 & $33.23 \pm 1.95$ \\
\hline Model & $32.52 \pm 2.85^{\mathrm{a}}$ & $26.85 \pm 2.31^{a}$ & $52.78 \pm 1.75^{a}$ & $123.57 \pm 7.73^{a}$ & $87.89 \pm 2.26^{a}$ & $50.93 \pm 1.82^{\mathrm{a}}$ & $42.99 \pm 1.25^{a}$ \\
\hline $\begin{array}{l}\text { Strychnine total } \\
\text { alkaloids high dose }\end{array}$ & $21.55 \pm 1.75^{b}$ & $21.96 \pm 1.04^{b}$ & $63.91 \pm 1.18^{b}$ & $100.38 \pm 2.48^{b}$ & $75.72 \pm 2.83^{b}$ & $32.62 \pm 2.35^{\mathrm{b}}$ & $38.57 \pm 0.72^{b}$ \\
\hline $\begin{array}{l}\text { Strychnine total } \\
\text { alkaloids medium dose }\end{array}$ & $21.32 \pm 2.38^{b}$ & $22.14 \pm 1.59^{b}$ & $64.72 \pm 1.70^{b}$ & $100.39 \pm 3.72^{b}$ & $75.64 \pm 2.99^{b}$ & $32.96 \pm 2.17^{b}$ & $38.52 \pm 1.33^{b}$ \\
\hline
\end{tabular}




\begin{tabular}{|c|c|c|c|c|c|c|c|}
\hline $\begin{array}{l}\text { Strychnine } \\
\text { alkaloids low dose }\end{array}$ & $24.45 \pm 2.48^{b}$ & $24.42 \pm 1.42^{\mathrm{b}}$ & $61.83 \pm 1.33^{b}$ & $106 \pm 3.34^{b}$ & $79.44 \pm 0.93^{b}$ & $38.18 \pm 0.90^{b}$ & $40.86 \pm 1.32^{b}$ \\
\hline Sodium hyaluronate & $21.21 \pm 2.03^{b}$ & $19.41 \pm 1.52^{b}$ & $65.93 \pm 1.99^{b}$ & $95.45 \pm 2.57^{b}$ & $73.02 \pm 1.84^{b}$ & $30.8 \pm 1.70^{\mathrm{b}}$ & $37.29 \pm 1.26^{b}$ \\
\hline
\end{tabular}

\section{Detection of IL-1, IL-6, SOD, LPO, NO, TNF in the synovial fluid and PYD in Urine by ELISA}

The expression of SOD in the synovial fluid was significantly decreased $(\mathrm{P}<0.05)$, and the expression of IL-1, IL-6, LPO, NO, TNF in the synovial fluid and PYD in urine in the osteoarthritic model group was significantly higher than that in the normal control group $(\mathrm{P}<0.05)$. Compared with the model group, the expression of IL-1, IL-6, LPO, NO, TNF in the synovial fluid and PYD in urine of the high, medium and low dose strychnine total alkaloid groups were significantly decreased $(\mathrm{P}<0.05)$. The expression of SOD in synovial fluid was similarly significantly increased $(\mathrm{P}<0.05)$ displaying a dose-dependent correlation. Likewise, the expression of IL-1, IL-6, LPO, NO, and TNF in the synovial fluid and PYD in urine of the sodium hyaluronate group was significantly decreased $(\mathrm{P}<0.05)$. Conversely, the expression of SOD in synovial fluid was significantly increased $(\mathrm{P}<0.05)$ in this group. When different total alkaloid dose groups were compared, expression of IL-1, IL-6, LPO, NO, and TNF in the synovial fluid and PYD in urine of the high and middle dose groups were significantly lower than those in the low dose group, demonstrating a dose-dependent correlation $(\mathrm{P}<0.05)$. Finally, no significant difference was observed at the level of IL-1, IL-6, SOD, LPO, NO, TNF and urine PYD in the middle and high dose of alkaloid and sodium hyaluronate group ( $\mathrm{P}$ $>0.05$ and Table 5).

\section{Discussion}

Intra-articular injection of drugs for the treatment of osteoarthritis has a history of $80 \mathrm{y}$ [2]. Intra-articular injection, as one of the main methods for clinical treatment of OA, has a characteristic of rapid effect. The treatment is simple, effective and easy to accept. The most representative injection was hyaluronic acid (HA) or corticosteroid which has been approved by United States Food and Drug Administration (US FDA) and European Medicines Agency (EMA) for the treatment of knee osteoarthritis (KOA). The clinical practice confirmed that the therapy can reduce joint pain and improve joint function, reduce the dosage of non-steroidal antiinflammatory drugs (NSAIDs) and delay the total knee replacement [3-7]. However, there are also problems such as short duration of curative effect, increased cost, and inappropriate use of patients in advanced stage and increased risk of infection in multiple articular cavities [8-11]. In recent years, the new therapies, such as the articular cavity injection of Platelet rich plasma (PRP) and mesenchymal stem cells (MSCs) appear which can improve the clinical symptoms of especially patients with the early osteoarthritis such as significantly reducing joint pain, promoting the articular cartilage repair and delay the degeneration of articular cartilage [12-14]. Few adverse reactions were reported $[12,15]$. However, its long-term efficacy and safety remains to be further observed [16].

Currently, the drugs used in the intra-articular injection are all western medicine. Osteoarthritis research society international (OARSI) of knee OA treatment guidelines issued in 2014, points out that the western medicine with certain curative effect in the improvement of clinical symptoms of OA can relieve pain and improve range of motion, but was unable to repair articular cartilage injury and could not stop the development of OA [17]. The treatment of osteoarthritis in Traditional Chinese Medicine mainly focuses on the protection of articular cartilage, which can delay articular cartilage degeneration and prevent further development of the disease [18-20]. Hence the model of Intra-articular injection Traditional Chinese Medicine for osteoarthritis is worth exploring. In this study, a papain model of rabbit knee osteoarthritis was established and the total alkaloids of Strychnosum were injected into joint cavity to explore the effects and mechanism of Strychnonine in order to provide the basis for its clinical application. The results showed that in the osteoarthritis model group the articular cartilage surface was not smooth, additionally, the cells in the superficial and radiation layers were markedly decreased. Moreover, the transitional layer cells were unevenly distributed and histopathological classification of the cartilage via Mankin's score was significantly higher than in the normal control group, indicating serious injury of the cartilage in the osteoarthritis knee joint. After treatment with the total alkaloids of Strychnine, the cartilage surface was smooth and the four-layer structure could be distinguished. Additionally, the number of chondrocytes was increased and the distribution was uniform. Consequently, Mankin's score was significantly improved, demonstrating that the total alkaloids of Strychnine can effectively repair cartilage injury of osteoarthritis.

Osteoarthritis is characterized by articular cartilage destruction and bone remodeling. While the pathogenesis is complex, at present, the pathogenic factors are largely considered to be inflammatory, free radicals and intraosseous pressure. The overexpression of a variety of inflammatory factors plays an important role in formation and development of osteoarthritis [21]. Among these inflammatory factors, IL-1, IL-6 and TNF- $\alpha$ are critical [22-25]. These cytokines disrupt articular cartilage by promoting the synthesis and release of matrix metalloproteinases, degrading cartilage matrix, inducing chondrocyte apoptosis and reducing extracellular matrix synthesis $[26,27]$. Our results showed that the expression of IL-1, IL-6 and TNF in the synovial fluid of the osteoarthritis model group increased a phenomenon that was mitigated after 
total alkaloid treatment in a dose-dependent manner. Therefore, the reparative effect of the total alkaloids of Strychnine on cartilage damage may effectively mediate the inhibition of IL-1, IL-6 and TNF expression during osteoarthritis.

Free radicals are also an important contributor to the pathogenesis of osteoarthritis. In the development of osteoarthritis, the body will produce free radicals, leading to oxidation/anti-oxidative imbalance, an imbalance which is critical in chondrocyte injury. Nitric oxide (NO) is an important free radical, playing an important role in cell to cell communication and antagonizing the oxidative damage of oxygen free radicals in the synovial fluid at low levels [28]. However, elevated synovial fluid NO may cause synovial inflammation, and increased inflammatory factors (such as IL-1, TNF) co-acting on articular cartilage, promoting articular cartilage degradation, and ultimately leading to bone inflammation [29]. Second, the free radical induced lipid peroxidation process can damage the endoplasmic reticulum, leading to bone collagen synthesis disorders and contributing to osteoarthritis. On the other hand, Superoxide dismutase (SOD) is an important free radical scavenger working toward the protection of synovial chondrocytes from damage. Unsurprisingly, the decreased activity of SOD promotes the degradation of articular cartilage, resulting in cartilage injury and promoting osteoarthritis [30,31]. Therefore, collective NO, lipid peroxidation (LPO) and SOD are important mediators of the pathogenesis of osteoarthritis. Accordingly, in patients with osteoarthritis, increased NO, increased LPO, decreased SOD and increased PYD could serve as indicators of the severity of osteoarthritis [32-37]. Among them, LPO as a metabolite of free radical lipid peroxidation, damaging the cell and cell membrane structure, can indirectly reflect the degree of damage caused by free radicals. Alternatively, pyridinoline (PYD) is a sensitive and specific biochemical marker of bone resorption which directly reflects the extent of bone resorption and the severity of osteoarthritis. In this study our results showed that the PYD in the urine of the osteoarthritis model group was predictably increased, while treatment with total alkaloids of Strychnine demonstrated a dose-dependent decrease, indicating through this biomarker the reparative potential of Strychnine alkaloids in osteoarthritis. On the other hand, the synovial NO and LPO of the model group were increased while SOD was decreased. Once again, treatment with the total alkaloids of Strychnine demonstrated through these markers the dose-dependent, restorative action of Strychnine. In sum, the reparative effect of the compound on osteoarthritic cartilage injury was closely related to the expression of these free radicals NO, LPO and SOD mediated by the total alkaloids of Strychnine.

In addition, bone hemodynamic abnormalities characterized by intraosseous venous stasis and the resulting intraosseous hypertension are also important factors leading to the development of osteoarthritis [38-40]. When bone internal venous stasis leads to high intraosseous pressure, risk of increased bone vascular dilation and diminished activity rises. In turn, the bone tissue may suffer from hypoxia and the accumulation of acid metabolites, resulting in "rest pain" and other clinical symptoms [41]. Therefore, the improvement of bone microcirculation and cartilage metabolism is of great significance to the prevention and treatment of osteoarthritis. The results of our study showed that while low, medium and high shear rate of blood viscosity and plasma viscosity were increased by osteoarthritis, treatment with the total alkaloids of Strychnine could alleviate blood and plasma viscosity. These results indicated that abnormal bone hemodynamics is an important factor leading to osteoarthritis, and revealed the total alkaloids can improve blood circulation disorders, reduce intraosseous pressure, pain and cartilage damage and play a role in the prevention and treatment of osteoarthritis.

In summary, inflammatory factors, free radicals, and intraosseous hypertension are important risk factors which are closely related with the occurrence and development of osteoarthritis. Strychnine total alkaloids have significant reparative effect on cartilage injury of osteoarthritis. Here we demonstrate that the mechanisms of action are multidimensional, including the inhibition of inflammatory factors such as IL-1, IL-6 and TNF, the regulation of free radicals such as NO, LPO and SOD and the improvement of cartilage metabolism. Collectively, the modulation of inflammatory factors and free radicals and improvement of bone microcirculation are important for the prevention and treatment of osteoarthritis. The therapeutic potential of strychnine in osteoarthritis warrants deeper investigation and application in future trials.

\section{Acknowledgement}

This project is supported by the key projects grant of Fujian Provincial Science and Technology Department (2012Y0039); this project is also supported by the Science Foundation of the Fujian Province, China (Grant No. 2017J01830).

\section{Conflict of Interest}

The authors declare no financial or commercial conflicts of interest.

\section{References}

1. Jun X, Qiang L, Xiaoliang S, Qingyuan Li. Effect of intraarticular injection of strychnine on cartilage injury in rabbit knee joint. Guide of China Medicine 11: 9-10.

2. Waugh WG. Acid Injection for osteo-arthritis. Br Med J 1945; 1: 873-874.

3. McCabe PS, Maricar N, Parkes MJ, Felson DT, O'Neill TW. The efficacy of intra-articular steroids in hip osteoarthritis: a systematic review. Osteoarthritis Cartilage 2016; 24: 1509-1517.

4. Matzkin EG, Curry EJ, Kong Q, Rogers MJ, Henry M, Smith EL. Efficacy and treatment response of intraarticular corticosteroid injections in patients with symptomatic knee osteoarthritis. J Am Acad Orthop Surg 2017; 25: 703-714.

5. Thomas T, Amouroux F, Vincent P. Intra articular hyaluronic acid in the management of knee osteoarthritis: 
Pharmaco-economic study from the perspective of the national health insurance system. PLoS One 2017 ;12: $\mathrm{e} 0173683$.

6. Ong KL, Anderson AF, Niazi F, Fierlinger AL, Kurtz SM, Altman RD. Hyaluronic acid injections in medicare knee osteoarthritis patients are associated with longer time to knee arthroplasty. J Arthroplasty 2016; 31: 1667-1673.

7. Delbarre A, Amor B, Bardoulat I, Tetafort A, PelletierFleury N. Do intra-articular hyaluronic acid injections delay total knee replacement in patients with osteoarthritisA Cox model analysis. PLoS One 2017; 12: e0187227.

8. Faúndez J, Cotoras $\mathrm{P}$, Irarrázaval S. Are intraarticular steroids effective for knee osteoarthritis? Medwave 2016; 16: e6599.

9. Weick JW, Bawa HS, Dirschl DR. Hyaluronic acid injections for treatment of advanced osteoarthritis of the knee: utilization and cost in a national population sample. J Bone Joint Surg Am 2016; 98: 1429-1435.

10. Chambers AW, Lacy KW, Liow MHL, Manalo JPM, Freiberg AA, Kwon YM. Multiple hip intra-articular steroid injections increase risk of periprosthetic joint infection compared with single injections. J Arthroplasty 2017; 32: 1980-1983.

11. Nguyen C, Rannou F. The safety of intra-articular injections for the treatment of knee osteoarthritis: a critical narrative review. Expert Opin Drug Saf 2017; 16: 897-902.

12. Fukawa T, Yamaguchi S, Akatsu Y, Yamamoto Y, Akagi R, Sasho T. Safety and efficacy of intra-articular injection of platelet-rich plasma in patients with ankle osteoarthritis. Foot Ankle Int 2017; 38: 596-604.

13. Yang X, Zhu TY, Wen LC, Cao YP, Liu C, Cui YP, Meng $\mathrm{ZC}$, Liu H. Intraarticular injection of allogenic mesenchymal stem cells has a protective role for the osteoarthritis. Chin Med J (Engl) 2015; 128: 2516-2523.

14. Kanwat H, Singh DM, Kumar CD, Alka B, Biman S, Aman H. The effect of intra-articular allogenic platelet rich plasma in Dunkin-Hartley guinea pig model of knee osteoarthritis. Muscles Ligaments Tendons J 2018; 7: 426-434.

15. Martini LI, Via AG, Fossati C, Randelli F, Randelli P, Cucchi D. Single platelet-rich plasma injection for early stage of osteoarthritis of the knee. Joints 2017; 5: 2-6.

16. Wehling P, Evans C, Wehling J, Maixner W. Effectiveness of intra-articular therapies in osteoarthritis: a literature review. Ther Adv Musculoskelet Dis 2017; 9: 183-196.

17. McAlindon TE, Bannuru RR, Sullivan MC, Arden NK, Berenbaum F, Bierma-Zeinstra SM, Hawker GA, Henrotin Y, Hunter DJ, Kawaguchi H, Kwoh K, Lohmander S, Rannou F, Roos EM, Underwood M. OARSI guidelines for the non-surgical management of knee osteoarthritis. Osteoarthritis Cartilage 2014; 22: 363-388.

18. Xu X, Lv H, Li X, Su H, Zhang X, Yang J. Danshen attenuates osteoarthritis-related cartilage degeneration through inhibition of NF- $\kappa \mathrm{B}$ signaling pathway in vivo and in vitro. Biochem Cell Biol 2017; 95: 644-651.
19. Zhong Y, Huang Y, Santoso MB, Wu LD. Sclareol exerts anti-osteoarthritic activities in interleukin-1 $\beta$-induced rabbit chondrocytes and a rabbit osteoarthritis model. Int J Clin Exp Pathol 2015; 8: 2365-2374.

20. Qin N, Wei L, Li W, Yang W, Cai L, Qian Z, Wu S. Local intra-articular injection of resveratrol delays cartilage degeneration in $\mathrm{C} 57 \mathrm{BL} / 6$ mice by inducing autophagy via AMPK/mTOR pathway. J Pharmacol Sci 2017; 134: 166-174.

21. Kapoor M, Martel-Pelletier J, Lajeunesse D, Pelletier JP, Fahmi H. Role of proinflammatory cytokines in the pathophysiology of osteoarthritis. Nat Rev Rheumatol 2011; 7: 33-42.

22. Attur M, Belitskaya-Lévy I, Oh C, Krasnokutsky S, Greenberg J, Samuels J, Smiles S, Lee S, Patel J, AlMussawir H, McDaniel G, Kraus VB, Abramson SB. Increased interleukin-1 $\beta$ gene expression in peripheral blood leukocytes is associated with increased pain and predicts risk for progression of symptomatic knee osteoarthritis. Arthritis Rheum 2011; 63: 1908-1917.

23. Larsson S, Englund M, Struglics A, Lohmander LS. Interleukin-6 and tumor necrosis factor alpha in synovial fluid are associated with progression of radiographic knee osteoarthritis in subjects with previous meniscectomy. Osteoarthritis Cartilage 2015; 23: 1906-1914.

24. Qu XQ, Wang WJ, Tang SS, Liu Y, Wang JL. Correlation between interleukin-6 expression in articular cartilage bone and osteoarthritis. Genet Mol Res 2015; 14: 14189-14195.

25. Livshits G, Zhai G, Hart DJ, Kato BS, Wang H, Williams FM, Spector TD. Interleukin-6 is a significant predictor of radiographic knee osteoarthritis: The Chingford Study. Arthritis Rheum 2009; 60: 2037-2045.

26. Ryu JH, Yang S, Shin Y, Rhee J, Chun CH, Chun JS. Interleukin-6 plays an essential role in hypoxia-inducible factor $2 \alpha$-induced experimental osteoarthritic cartilage destruction in mice. Arthritis Rheum 2011; 63: 2732-2743.

27. Fernandes JC, Martel-Pelletier J, Pelletier JP. The role of cytokines in osteoarthritis pathophysiology. Biorheology 2002; 39: 237-246.

28. Vuolteenaho K, Moilanen T, Hämäläinen M, Moilanen E. Regulation of nitric oxide production in osteoarthritic and rheumatoid cartilage. Role of endogenous IL-1 inhibitors. Scand J Rheumatol 2003; 32: 19-24.

29. Rui L, Chang G, Yongtiao H, Huichun Z. The role of NO, TNF- $\alpha$ in osteoarthritis. J Radioimmunol 2011; 24: 351-352.

30. Ruiz-Romero C, Calamia V, Mateos J, Carreira V, Martínez-Gomariz M, Fernández M, Blanco FJ. Mitochondrial dysregulation of osteoarthritic human articular chondrocytes analyzed by proteomics: a decrease in mitochondrial superoxide dismutase points to a redoximbalance. Mol Cell Proteomics 2009; 8: 172-189.

31. Gavriilidis C, Miwa S, von Zglinicki T, Taylor RW, Young DA. Mitochondrial dysfunction in osteoarthritis is associated with down-regulation of superoxide dismutase 2 . Arthritis Rheum 2013; 65: 378-387. 
32. Scher JU, Pillinger MH, Abramson SB. Nitric oxide synthases and osteoarthritis. Curr Rheumatol Rep 2007; 9: 9-15.

33. Pinto S, Rao AV, Rao A. Lipid peroxidation, erythrocyte antioxidants and plasma antioxidants in osteoarthritis before and after homeopathic treatment. Homeopathy 2008; 97: $185-189$.

34. Regan E, Flannelly J, Bowler R, Tran K, Nicks M, Carbone BD, Glueck D, Heijnen H, Mason R, Crapo J. Extracellular superoxide dismutase and oxidant damage in osteoarthritis. Arthritis Rheum 2005; 52: 3479-3491.

35. Scott JL, Gabrielides C, Davidson RK, Swingler TE, Clark IM, Wallis GA, Boot-Handford RP, Kirkwood TB, Taylor RW, Young DA. Superoxide dismutase downregulation in osteoarthritis progression and end-stage disease. Ann Rheum Dis 2010; 69: 1502-1510.

36. Garnero P, Piperno M, Gineyts E, Christgau S, Delmas PD, Vignon E. Cross sectional evaluation of biochemical markers of bone, cartilage, and synovial tissue metabolism in patients with knee osteoarthritis: relations with disease activity and joint damage. Ann Rheum Dis 2001; 60: 619-626.

37. Seibel MJ, Duncan A, Robins SP. Urinary hydroxypyridinium crosslinks provide indices of cartilage and bone involvement in arthritic diseases. J Rheumatol 1989; 16: 964-970.
38. Arnoldi CC, Djurhuus JC, Heerfordt J, Karle A. Intraosseous phlebography, intraosseous pressure measurements and 99mTC-polyphosphate scintigraphy in patients with various painful conditions in the hip and knee. Acta Orthop Scand 1980; 51: 19-28.

39. Junxing Y, Shucheng B, Luhong J. The effect of Gucixiaotong capsule on high knee intraosseous pressure and hemorheology of rabbit. Chin J Trad Med Traumatol Orthop 2007; 15: 45-48.

40. Mapp PI, Sagar DR, Ashraf S, Burston JJ, Suri S, Chapman V, Walsh DA. Differences in structural and pain phenotypes in the sodium monoiodoacetate and meniscal transection models of osteoarthritis. Osteoarthritis Cartilage 2013; 21: 1336-1345.

41. Lili Q, Haidong W, Anguo L. Treatment of 35 cases of degeneration of knee joint with closed decompression and percutaneous operation. Chin J Trad Med Traumatol Orthop 2016; 24: 29-32.

\section{${ }^{*}$ Correspondence to}

Zhenqiang Hong

College of Traditional Chinese Medicine

Fujian University of Traditional Chinese Medicine

PR China 